\title{
SYSTEM COMPOSER: Technology for Rapid System Integration and Remote Collaboration*
}

\author{
Brady R. Davies and Robert D. Palmquist \\ Sandia National Laboratories \\ P.O. Box 5800 MS 1176 \\ Albuquerque, New Mexico 87185-1176
}

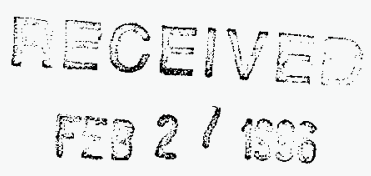

\begin{abstract}
Sandia National Laboratories has developed an approach to the design, evaluation, deployment and operation of intelligent systems which is called System Composer. This toolkit provides an infrastructure and architecture for robot and automation system users to readily integrate system components and share mechatronic, sensor, and information resources over networks. The technology described in this paper provides a framework for real-time collaboration between researchers, manufacturing entities, design entities, and others without regard to relative location. An overview of the toolkit including its elements and architecture is provided along with examples of its use.
\end{abstract}

Table 1 contains a list of all acronyms and their descriptions as used in this paper.

\section{INTRODUCTION}

As networking technologies have continued to improve and proliferate, the ability to share, not only information, but also mechatronic assets, has become possible (in this paper "mechatronic" resources refers to electromechanical devices with computer interfaces). Timely and efficient collaboration between entities has become increasingly important as corporations form technology alliances in response to global competition and markets[1]. This ability to quickly share and collaborate on systems, software, and mechatronic devices owned or operated by disparately located entities may soon prove to be a crucial business advantage. The System Composer toolkit and the associated technologies described in this paper provide the environment for flexible and efficient integration, interaction, and information exchange between disparate entities to occur, thus making remote resource sharing viable.

Some similarities exist between the System Composer remote access tools and remote machine access technologies developed at other institutions such as the Jet Propulsion Laboratory (JPL) and the National Aeronautics and Space Administration (NASA). JPL and NASA have developed technology to teleoperate devices located in space or other very remote environments. These types of systems have very specialized interfaces and interconnections and rely heavily on human-in-the-loop control techniques. Sandia's system incorporates generic interfaces, system reconfigurability, graphical programming, and automatic programming.

System Composer's architecture is based on emerging network communication technologies and two Department of Energy developed technologies called Technology Information Environment for Industry (TIE-In), a gateway server technology for securely operating software over computer networks [2], and Generic Intelligent System Controller (GISC), a library of software modules used to configure control systems and communications between computers and mechatronic devices [3]. The architecture facilitates design, evaluation, deployment and operation of multiple intelligent programmable machines (like robots, machine tools, etc.), input devices (like computer mice, force/torque reflective feedback devices, sensors, etc.), software tools (like simulation code, control algorithms, graphical models, etc.), and sensors, from local and/or remote locations.

An existing prototype architecture and system

* This work was performed at Sandia National Laboratories supported by the United States Department of Energy under contract number DE-AC04-94AL85000. 


\section{DISCLAMMER}

Portions of this document may be illegible in electronic image products. Images are produced from the best available original document. 
has been developed and demonstrated at Sandia National Laboratories in Albuquerque, New Mexico, Control of Sandia robots using these technologies has also been demonstrated from such remote locations as Washington D.C., California, and Michigan. An enhanced version that includes secure access and is compatible with more cost-effective hardware and software is currently being implemented. This secure capability for sharing mechatronic resources over computer networks will be described in a later section of this paper

\section{SYSTEM PHILOSOPHY}

The System Composer philosophy truly embraces a "systems engineering" approach to integration and collaboration. The technology provides the capability to integrate both real and virtual devices (like sensors, robots, machine tools, etc.) into the same environment and provides a seamless interchange between the virtual and real environments[4]. For example, if a robot module is selected as virtual, then an appropriate simulated robot, typically a softwaremodule routine running on a Virtual-ModuleEuropa (VME) system, is automatically connected to the system. If instead the real robot is selected, then the system automatically reconfigures itself so that the actual robot is controlled. This applies not only to robots but also to any component within the system. For example, a virtual sensor subsystem may be placed on a real robot or likewise a real sensor may be placed on a virtual robot. What enables this is that the subsystem interfaces for both the virtual and actual components are identical, thus the entire integration, from operator interface to path planners to sensors can be configured, operated and evaluated prior to actually procuring the real robot. Once the real robot becomes available, then the same software that was used to drive the simulation is used to drive the actual device. This combined with the ability to seamlessly shift from virtual devices to actual devices provides the means to accurately evaluate and thereby reduce the risks associated with systems design, procurement and deployment. The correlation between the virtual and real environments can be further improved by adding appropriate sensors.

As was previously mentioned, the software tools within System Composer are all defined as interchangeable modules. An important feature is the ability to add new modules without reconfiguring the entire system. This simplifies the incorporation, testing, and enhancement of new technology approaches by users. An experimenter only provides the increment of technology needed to test a new concept or approach. This reduces the time and cost of technology development while stimulating cross institutional teaming. Further, by using this modular approach, the system designer need only consider the functional characteristics of the system modules (the "systems" approach). Likewise the module developers need not consider application specific details. This development environment enables the library of modules to be continually expanded and reconfigured as necessary.

\section{TECHNOLOGY DESCRIPTION}

Included in System Composer is a set of system integration technologies which provide modular reconfigurability of multiple devices using a library of software tools. The devices that can make up a system include mechatronic components such as sensors, software tools, input devices, etc. The architecture encompasses an environment where modules associated with various hardware and software devices can be readily reconfigured, modified, and expanded.

System Composer utilizes Graphical User Interface (GUI) tools to simplify integration of new supervisor or subsystem elements. These GUI tools utilize the graphics libraries of the host graphics workstation and commercially available GUI tool software libraries. Different software displayed "buttons" are used to select, connect, layout, verify, and even review software process descriptions of various supervisors and subsystems like computing resources, input devices, sensors, robots, etc. [5].

Various categories of resources can be implemented including (but not limited to): robots, operator interfaces, sensors, product expert systems, process expert systems, controls, machine tools, assembly systems, and communications. These resources are available to users as modules and are represented as graphical icons in System Composer. 
Communication and control between modules is made possible through the use of client/server interfaces for generic interconnectability[3], a passive control approach for inherent system stability [6], and agents for rapid reconfigurability of multiple device systems. These technologies are described in the following sections.

\section{A. Communication Interfaces}

The communication interfaces within System Composer use a Client/Server approach where software drivers translate generic commands into specific instructions required by each subsystem. By defining the driver interface, virtual models of the device communicate in the same manner as the actual hardware. Clients and Servers are connected through communication links using either UNIX sockets or the Sandia developed GENeral Interface for Supervisor and Subsystems (GENISAS)[3].

The communications links support both timebased and event-based control methods. An example of a time-based control element is a joy stick. Using a joy stick as an input device, the user interacts with the subsystem in real time, entering tasks for the subsystem to perform. The subsystem responds immediately to user commands. An example of an event-based control element is a task planner. A task planner specifies the sequence of operations required to achieve a given task. In this case, the complete sequence of events is described prior to initiating any operations and the completion of prior events initiates subsequent events. Both of these supervisor types can be plugged into the system simulation as needs dictate.

Subsystems receive and execute commands from supervisor agents. These subsystems are categorized into classes of mechatronic components such as robots, manufacturing equipment, intelligent end-effectors, vision systems, etc. Each subsystem resource is completely characterized by three attributes: hardware type, behavior mode and capabilities list. Hardware Type includes information such as device model and type, and includes computing resources or sensors associated with the device. Behavior Mode describes the operational behavior of the agent. For example, a subsystem such as a robot could be configured with several behavior modes including operating in an autonomous mode under sensor control, in a teleoperation mode with various input control devices such as a force reflecting master, or even in a programmable mode using high-level decision planners. Finally, the Capabilities List specifies the type of operations that the particular system can perform. Examples of items in this list are commands such as "get_tool", "move_home", and "move_along_path".

These three attributes also define the respective interface requirements: Hardware Type dictates mechanical and electronic coupling or connection, Behavior Mode dictates types of $\mathrm{I} / \mathrm{O}$ coupling and information flow direction, and the Capabilities List dictates the logical operation command set.

\section{B. Secure Communications}

The Technology Information Environment for Industry (TIE-In) is a new remote electronic access technology being implemented by Sandia. TIE-In enables gateway service that provides access between a single server receiving data packets on a designated port and any one of a set of remote systems needing to send information to that server. The gateway is created by way of a separate gateway client. This client controls which servers and clients are able to exchange data. The gateway service then establishes a new server whose function is to accept information from authorized senders and forward the information to the appropriate receiving servers[2].

TIE-In provides rigorous secure access protection for both remote and local system users. This is important to protect hardware from unauthorized operation and proprietary data from competitive disclosure. While TIE-In somewhat modifies the rate of information transfer over computer networks, Sandia has successfully operated full video conferencing bandwidths through the server with minimal reduction in video quality and speed.

\section{Control Behavior}

As was previously mentioned, System 
Composer uses a passive control theory approach which ensures stable controller configurations. This approach, Sequential Modular Architecture for Robotics and Teleoperation, known as SMART[6] is coupled with the Robot Independent Programming Language, known as RIPL[7] to build the behavior modes of subsystems. SMART provides a real-time control architecture for constructing telerobotic systems consisting of a multitude of classes such as robots, sensors, input and output devices, etc. SMART modules can run synchronously and can be distributed arbitrarily across multiple CPUs connected across networks. A robot server, created with the RIPL, is connected via a network to a supervisor resource, thus completing the communications path from the supervisor to SMART. Using these software packages, it is possible to build and reconfigure flexible robot servers for various robots incorporating different input devices and sensors operating over networks.

\section{REMOTE ACCESS}

The hardware aspects of production, manufacturing, or processing systems are very expensive. If system flaws can be identified in the design phase of a project, a significant amount of time and money can be saved during the implementation and operation phases. A testbed (A 100 pound payload, 20' $\mathrm{x} 40^{\prime}$ workspace gantry robot with graphical programming and video conferencing user interface software/hardware systems) was developed to evaluate these technologies for verifying and validating designs prior to procurement of hardware and for the sharing of mechatronic resources.

The testbed system provides connectivity between remote computer workstations, local computer workstations, robot controllers, video systems, video controllers, robot subsystem controllers, and various graphics and video display systems.

Local networks are connected via high-speed communications links (ethernet). Network connections between the remote user network and Sandia's local network are through a dedicated communications link.
As the Sandia testbed is currently configured, a remote user requires the use of two computer workstations: one workstation to handle all video conferencing and video interfacing and another to serve as the user interface between the robot and other mechatronic devices. The user interface between the robot and other mechatronic devices is configured as a graphicsbased control system. Interaction is provided to the user through a GUI tool that allows the user to quickly and easily program, control, monitor, test, and configure the robot and other mechatronic devices.

In the System Composer environment, a graphical representation of the robot and its environment are modeled and displayed using robot simulation software and a graphics workstation. A graphics workstation, running simulation and display software, functions as the primary user interface for programming, commanding, and otherwise interacting with the actual robot. Simulation software is used to display the world model in real-time for operator validation of robot motion. It is also used to visually preview robot motion in order to validate safe operation and verify correct task performance prior to actual robot motion[8]. The simulation can also be configured to perform mathematical automatic collision checking and safe path verification.

Servo control is performed local to the machine as an intelligent subsystem. High speed sensing subsystems provide the needed sensory inputs to a subsystem's motion control algorithms to perform, for example, force controlled interactions with the environment. Real-time sensing also allows in-process modification of machine motions. To minimize the effects of network latencies, high speed sensor-based servo-controlled operations are executed within the subsystem controller and not by the higher level GISC supervisor.

In typical user sessions, tasks were selected and defined by the remote user. An automated planning and programming system generated robot motion plans needed to execute the task, and the user was able to accept or reject plans. Once a plan was accepted, it was transmitted to the Sandia gantry robot. The graphics system updated the graphical robot position using actual 
robot position encoder data to the remote user to monitor the system. Real-time video images of the actual robot were also transmitted to the remote user via the teleconferencing system.

\section{SYSTEMS INTEGRATION}

A number of other systems have been configured and operated using System Composer. In one such configuration, the primary user site consisted of three VME computing subsystems dedicated to system resource software and a graphics workstation that functioned as the primary user interface displaying graphical simulations of workcell hardware and various control and status menus. Input devices at the primary site included GUI interfaces, a force reflecting torque arm, and a spaceball threedimensional joystick.

Both a virtual robot and a real robot were integrated into the system. The real robot was located in a remote lab (approximately one mile from the primary user site) and connected to the primary user site via an ethernet network. The virtual robot was part of the graphics simulation located at the primary site. A capacitance based proximity sensor was integrated into the system at a third location. A video conferencing system was used to provide live feedback from the remotely located real robot to the primary user site.

The system was constructed by first implementing the virtual robot on the VMEbus computing system and adding the capacitancebased proximity sensor via the network connection. This enabled the system performance of the sensor to be evaluated prior to integrating the actual robot into the system. Next, the force reflecting torque arm and spaceball were connected to the system over the network. With the torque arm operational, the operator could literally feel either virtual forces generated by the virtual robot or virtual forces created from transduced real sensor measurands from the proximity sensor.

This configuration provided an efficient means for accomplishing many different tasks: evaluating the system prior to hardware commitment, training operators, and programming the controls and communications of the system. Once the system was completed, the real robot was integrated with the same software and input devices used in the virtual configuration state.

Using System Composer, the integration process took a single user less than ten minutes to implement (not including component module development time). Without System Composer it is estimated that the integration time would have exceeded two months (this includes all software development and integration implementation) [9]. With System Composer, once a module for a particular resource has been defined, it need not be redefined or recreated. It is simply added to the System Composer library and reused by other users through network access.

In another application, System Composer was used to integrate a flexible assembly cell for electro-mechanical assemblies. This assembly cell, called the Agile Manufacturing Production System (AMPS), includes three AdeptOne SCARA robots, a Staubli RX-90 robot, a Bosch conveyor system, vision systems, and part trays. The production script or control logic was automatically derived from a CAD file by a Sandia-developed expert assembly system called Archimedes. Using System Composer, a virtual representation of the assembly system was integrated and tested in less than twenty percent of the estimated time that would be required to implement the system in simulation [9].

Using System Composer, the AMPS workcell could have alternatively been configured and tested with some or all of the equipment components located at different sites. Then the various equipment components could be collocated and integrated more quickly and efficiently. To accomplish this, a complete virtual representation of the workcell would first be constructed. Control and communication software would then be developed, integrated, and tested in the virtual workcell. Once the virtual workcell was operating properly, the actual disparately located equipment components would be connected using network connections to transmit and receive control and data information. Final testing and "fine tuning" would likely be required to facilitate differences between the virtual and real systems attributed to modeling errors, network latency and signal speed deviations, mechanical interactions between equipment components, etc. 


\section{SUMMARY}

System Composer technology enables diverse users to easily synthesize and share mechatronic resources and systems. Integration of components into systems is accomplished by simply connecting predefined modules. An integral information architecture transmits and uses information to automate programming of machines. Further enhancements to the technologies will facilitate access to other mechatronic resources by an increasingly greater scope of remote users. The use of this technology provides greater access to expensive capital equipment, facilitates rapid prototyping, and shorten the product-to-market cycle.

\section{ACKNOWLEDGMENTS}

The authors thank Robert Anderson, Scott Gladwell, Michael McDonald, Fred Oppel, and Dan Small for their significant contributions to this research.

\section{REFERENCES}

[1] Design News Staff, "Engineering Megatrends", Design News, August 28, 1995, pp. 59-78.

[2] "TIE-In Internet Protocol Gateway", an internally generated white paper at Sandia National Laboratories, 1995.

[3] Griesmeyer, J. M., M. J. McDonald, R. W. Harrigan, P. L. Butler, and J. B. Rigdon, "Generic Intelligent System Control (GISC)", Sandia Internal Report, SAND92-2159, 1992.

[4] Oppel, F. J., R. D. Palmquist, and B. R. Davies, "Remote Access and Modular Integration of Mechatronic Resources Using Virtual Collaborative Environments (VCE) and the Distributed Collaborative Workbench (DCW)", ARPA Workshop white paper, March, 1995.

[5] Palmquist, R. D., F. J. Oppel, III, and B. R. Davies, "Systems Toolkit for Configuring and Integrating Intelligent Systems", Proceedings of the SPIE International Symposium on Intelligent Systems and Advanced Manufacturing, Oct. 22-26, 1995.
[6] Anderson, R. J., "SMART: A Modular Architecture for Robotics and Teleoperation", International Symposium on Robotics and Manufacturing (ISRAM '93), Santa Fe, NM, April, 1993.

[7] Miller, D. J. and R. C. Lennox, "An ObjectOriented Environment for Robot System Architectures", Control Systems, IEEE, Vol. 11, No. 2, 1991.

[8] McDonald, M. J. and R. D. Palmquist, "Graphical Programming: On-Line Robot Simulation for Telerobotic Control", International Robots and Vision Automation Show and Conference Proceedings, pp. 22-59 to 22-73, 1993.

[9] Bennett, P. C., "Rapid Assembly and Use of Robotic Systems: Saving Time and Money in New Applications", to be published in the Proceedings of Emerging Technologies and Applications in Remote Handling and Robotics, 1996.

\begin{tabular}{|l|l|}
\hline Acronym & \multicolumn{1}{c|}{ Definition } \\
\hline JPL & Jet Propulsion Laboratory \\
\hline NASA & $\begin{array}{l}\text { National Aeronautics and } \\
\text { Space Administration }\end{array}$ \\
\hline TIE-In & $\begin{array}{l}\text { Technology Information } \\
\text { Environment for Industry }\end{array}$ \\
\hline GISC & $\begin{array}{l}\text { Generic Intelligent System } \\
\text { Controller }\end{array}$ \\
\hline VME & Virtual-Module-Europa \\
\hline GUI & Graphical User Interface \\
\hline GENISAS & $\begin{array}{l}\text { GENeral Interface for } \\
\text { Supervisor and Subsystems }\end{array}$ \\
\hline SMART & $\begin{array}{l}\text { Sequential Modular } \\
\text { Architecture for Robotics } \\
\text { and Teleoperation }\end{array}$ \\
\hline RIPL & $\begin{array}{l}\text { Robot Independent } \\
\text { Programming Language }\end{array}$ \\
\hline AMPS & $\begin{array}{l}\text { Agile Manufacturing } \\
\text { Production System }\end{array}$ \\
\hline
\end{tabular}

Table 1: Acronym Definitions 


\section{DISCLAIMER}

This report was prepared as an account of work sponsored by an agency of the United States Government. Neither the United States Government nor any agency thereof, nor any of their employees, makes any warranty, express or implied, or assumes any legal liability or responsibility for the accuracy, completeness, or usefuiness of any information, apparatus, product, or process disclosed, or represents that its use would not infringe privately owned rights. Reference herein to any specific commercial product, process, or service by trade name, trademark, manufacturer, or otherwise does not necessarily constitute or imply its endorsement, recommendation, or favoring by the United States Government or any agency thereof. The views and opinions of authors expressed herein do not necessarily state or refiect those of the United States Government or any agency thereof. 